\title{
Avaliação prévia da toxicidade de um efluente simulado derivado de petróleo sobre Promysis atlantica (Crustacea, Mysidacea)
}

\author{
PHAN Van Ngan; Vicente GOMES \& Maria José de Arruda Campos Rocha PASSOS
}

Instituto Oceanográfico da Universidade de São Paulo

(Caixa Postal 66149, 05389-970, São Paulo, SP, Brasil)

- Abstract: Preliminary experiments were carried out to estimate the potential toxicity of an effluent derived from petroleum to be dumped into São Sebastião channel through a submarine emissary which is being built at the Maritime Terminal "Almirante Barroso" (TEBAR). Two simulated samples of this effluent (denominated A and B) were supplied by PETROBRÁs. The toxicity of sample A was tested with young and adult Promysis atlantica (Crustacea, Mysidacea) and the toxicity of sample B only with young individuals of the same species. In relation to Effluent-A, concentrations between $1 \%$ and $5 \%$ increase significantly the rate of mortality of animals in short term tests $(24-96 \mathrm{hrs})$. The results also indicated that, in this case, adults are less resistant than youngs. Concentrations of $0.075 \%$ of Effluent-B are at the maximum limits of no effect observation for short term expositions of young animals ( $96 \mathrm{hrs})$. The two effluent samples are different from each other. Both of them are very toxic, but the second is about 10 times more toxic than the first one. The toxicity of the samples also varies in function of time of storage. It seems that this variation is not predictable as the results indicated that the toxicity increased with storage for sample A and diminished for sample B. Other possible effects of the effluent on the ecosystem are discussed.

- Resumo: Foram realizados experimentos para avaliar previamente o potencial tóxico de duas amostras de efluente derivado de petróleo a ser lançado no canal de S. Sebastiāo, através de um emissário submarino que está sendo construído no Terminal Marítimo "Almirante Barroso" (TEBAR). As amostras (denominadas Efluente-A e -B) foram fornecidas pela PETROBRAS e foram testadas com jovens e adultos de Promysis atlantica (Crustacea, Mysidacea). Em relação ao Efluente-A, concentraçōes entre $1 \%$ e $5 \%$ alteram significativamente a taxa de mortalidade dos animais em curto intervalo de tempo (24-96 horas). No caso do Efluente-B concentraçōes de $0,075 \%$ (96 horas) estão no limite máximo de exposição que não afeta a sobrevivência dos jovens da mesma espécie. Os testes demonstraram serem as duas amostras (Efluentes A e B) bastante diferentes uma da outra, sendo a segunda cerca de dez vezes mais tóxica que a primeira. Além disso, a toxicidade dos efluentes varia também em funçāo do armazenamento. Outros possíveis efeitos dos efluentes sobre o ecossistema sāo discutidos.

- Descriptors: Mysidacea, Promysis atlantica, marine toxicology, toxicity, petroleum.

- Descritores: Mysidacea, Promysis atlantica, toxicologia marinha, toxicidade, petróleo.

\section{Introdução}

Os problemas toxicológicos marinhos mais urgentes do momento atual, exigem a inclusão do estabelecimento de critérios e a padronização de métodos de controle da poluição visando, principalmente, estabelecer os limites máximos aceitáveis de poluentes na água do mar e prever e avaliar as possíveis conseqüências biologicas destes poluentes nos ecossistemas.

O estudo sobre os efeitos de poluentes no ecossistema como um todo é extremamente complexo e por vezes, inviável devido a diversos fatores tais como custos, disponibilidade de tempo, extensão das áreas sob impacto e diversidade das espécies envolvidas. Entretanto, para poder estimar os efeitos deletérios de materiais tóxicos sobre o meio ambiente é freqüentemente necessário 
obterem-se respostas rápidas. Nesse sentido, os testes de toxicidade aguda, são ferramentas importantes e confiáveis para estimar as concentraçōes nas quais um determinado produto tóxico provoca efeitos deletérios em uma dada população de organismos selecionada (Ward \& Parrish, 1982; Capuzzo et al., 1988). Para a realizaçāo destes testes, a escolha dos organismos deve obedecer a uma série de critérios que dependem do propósito do experimento e das características das èspécies (Craddock, 1977; Bellan, 1981).

Os misidáceos são crustáceos que se mostram tão ou mais sensíveis à exposiçāo a substâncias tóxicas do que outras espécies já testadas. Sua facilidade de manuseio e cultivo, ciclo de vida curto, pequeno tamanho e desenvolvimento larval direto, fazem deles organismos apropriados para experimentos de bioensaio. Por sua semelhança biologica com as formas juvenis de outras espécies da mesma classe, são também muito utilizados para se avaliar o impacto de poluentes em crustáceos de importância comercial como camarōes, lagostas e caranguejos (Nimmo \& Hamaker, 1982). Os misidáceos ocupam um lugar importante no ecossistema marinho, pois constituem um dos componentes da dieta de várias espécies de peixes, muitas utilizadas para o consumo humano (Evans et al., 1982). Como os peixes acumulam em seus tecidos os produtos tóxicos advindos do alimento, qualquer contaminação dos misidáceos pode trazer sérias conseqüências para o ecossistema. Além disso, devido ao comportamento de forte migração vertical da maioria das espécies, os misidáceos possuem um potencial de rápido transporte de tóxicos da superfície para os sedimentos marinhos e vice-versa (Evans et al., op. cit.).

Promysis atlantica (Tattersall, 1923), a espécie empregada neste trabalho, reúne todas as características acima citadas que fazem dos misidáceos organismos altamente recomendáveis para os testes de toxicidade. Além disso, ocorre abundantemente em Ubatuba, local onde se situa a Base Norte de pesquisa do Instituto Oceanográfico da USP.

A PETROBRÁS pretende construir no Terminal Marítimo "Almirante Barroso" (TEBAR) um emissário submarino para lançar no canal de São Sebastião (litoral Norte de São Paulo) um efluente aquoso derivado de petróleo com composiçāo complexa. Antes que se inicie seu lançamento, foi realizado através de um convênio entre a PETROBRÁS, a FUNDESPA - Fundação de Estudos e Pesquisas Aquáticas e o Instituto Oceanográfico da USP, um projeto multi-disciplinar para estudar possíveis impactos ambientais e também, para verificar a eficiência e a reprodutividade do tratamento que se pretende realizar antes do despejo. Este trabalho faz parte desse projeto e tem como objetivos avaliar previamente a toxicidade daquele efluente sobre Promysis atlantica, um crustáceo do zooplâncton.

\section{Material e métodos}

\section{Enuente}

Foram testadas duas amostras, por nós denominadas de Efluente-A e Efluente-B, com o objetivo de comparar seus potenciais tóxicos. Elas foram preparadas pela PETROBRÁS, em dias distintos, misturando-se, nas proporçōes necessárias, os resíduos que farāo parte do efluente real. Estes incluem compostos das drenagens de águas contaminadas das áreas de tanques, válvulas e bombas, água de lastro e de produtos, assim como compostos provenientes da dessalgação do petróleo. Ambas foram tratadas pelos técnicos da empresa para redução dos compostos feńlicos e dos sulfetos, segundo métodos que deverão fazer parte da rotina de tratamento do efluente. As amostras foram transportadas em botijōes de polietileno branco acondicionados em caixas de isopor com gelo, para o laboratório em São Paulo, onde foram mantidas em câmara frigorífica a $0^{\circ} \mathrm{C}$, até o início dos testes.

O Efluente-A foi amostrado no dia 25/11/91 e empregado em 5 experimentos; o Efluente-B foi amostrado no dia $06 / 01 / 92$, sendo empregado em 2 experimentos. Foram realizadas análises de DBO, DQO, bleos e graxas, sulfetos e fenóis de ambos os efluentes segundo a metodologia descrita em "Standard Methods for the Examination of Water and Wastewater" (1985).

\section{Coleta e manutenção dos animais}

Foram empregados nos experimentos com o Efluente-A, jovens e adultos do misidáceo Promysis atlantica, e nos experimentos com o Efluente-B, apenas jovens dessa espécie. Os animais foram coletados em Ubatuba, em bancos de algas sobre fundo rochoso defronte à Base Norte do Instituto Oceanográfico da USP. Estes foram capturados com auxilio de um recipiente plástico de 2 litros, que possui uma abertura recortada lateralmente, recoberta com uma tela fina de $315 \mu \mathrm{m}$. A coleta é feita mergulhando-se o recipiente na água sendo que o excesso escorre pela abertura da jarra. A tela impede o escape dos espécimens. Dessa forma procurou-se reduzir ao máximo os traumas físicos e fisiologicos decorrentes da coleta. Para o transporte, os animais foram acondicionados em sacos plásticos de 5 litros de capacidade, preenchidos com 3 litros de água do mar filtrada, cada um com aproximadamente 100 animais. Uma vez preparados, os sacos plásticos foram insuflados com oxigênio puro, para que houvesse troca de oxigênio entre o ar e a água, à medida que este fosse sendo consumido. Testes realizados há vários anos em nosso laboratório demonstraram a eficiência do procedimento acima descrito. 
Em São Paulo os animais foram mantidos em aquários com água do mar filtrada, em sala com temperatura controlada entre 22 e $23^{\circ} \mathrm{C}$. Os jovens foram alimentados com náuplios de Artemia salina recém-eclodidos e os adultos com náuplios de até 96 horas.

\section{Procedimentos para os experimentos}

Os experimentos foram elaborados baseando-se na metodologia descrita por Bellan (1981), Ward \& Parrish (1982) e Anderson et al. (1988).

Os experimentos foram de curta duração, de no máximo 96 horas, sem renovação do meio (estáticos), com ciclo de luz natural, em temperatura constante de $22-23^{\circ} \mathrm{C}$. Cada experimento consistiu na exposição dos animais a diversas concentraçöes dos efluentes, diluídos em água do mar filtrada em filtro (CUNO) com porosidade de $2 \mu \mathrm{m}$, coletada nas proximidades da Ilha Anchieta, em Ubatuba, com salinidade de $36 \%$ a $38 \%$. Essa mesma água, sem qualquer diluição, foi utilizada nos frascos controle. Os efluentes são hipersalinos (67\% para o Efluente-A e $62 \%$, para o Efluente-B). Para eliminar os efeitos da salinidade, foi utilizada água destilada para ajustar a salinidade das diluiçōes ao nível daquela do controle, sem alterar as concentraçōes do efluente nas diluiçōes desejadas, conforme a seguinte formula:

$$
V d=\frac{V e x(S e-S m)}{S m}
$$

onde

$\mathrm{Vd}=$ volume de água destilada $\mathrm{em} \mathrm{ml}$ a ser adicionada;

$\mathrm{Ve}=$ volume do efluente necessário $\mathrm{em} \mathrm{ml}$ para preparar um litro de uma diluiçāo com uma determinada concentraçāo;

$\mathrm{Se}=$ salinidade do efluente;

$\mathrm{Sm}=$ salinidade da água do mar;

completada até um litro com água do mar.

Experimentos preliminares demonstraram que a adição de água destilada utilizada no ajuste da salinidade, nāo afeta a qualidade da água do mar e nem a sobrevivência dos animais.

Cada experimento consistiu de controle com 5 a 10 réplicas, cada réplica contendo 10 animais, totalizando de 50 a 100 animais; e de diversas diluiçōes previamente selecionadas, cada uma com 3 a 5 réplicas, cada réplica contendo 10 animais, totalizando, desse modo, 30 a 50 animais por diluição. Cada réplica continha $250 \mathrm{ml}$ de solução colocados em béquer de $400 \mathrm{ml}$. Devido ao volume de água em relaçāo ao número de animais e o período experimental, não houve necessidade de aeração artificial.
Além disso, a aeração poderia alterar mais rapidamente a composição da diluição pela volatilização de componentes do efluente (Craddock, 1977).

Foram realizados experimentos com jovens e adultos. Os jovens utilizados nos experimentos foram aqueles liberados no laboratório em um único dia, tendo portanto, a mesma idade. Os adultos foram somente aqueles sem jovens no marsúpio e com tamanhos aproximadamente uniformes. A contagem dos sobreviventes era realizada a cada 24 horas.

Nos experimentos acima de 24 horas, os indivíduos mortos eram retirados após a contagem. Os sobreviventes eram alimentados com Artemia salina em quantidade suficiente para que não sobrassem indivíduos nāo consumidos no dia seguinte (cerca de $\mathbf{4 0}$ artemias por animal). Caso o fundo do béquer apresentasse detritos, estes eram removidos com pipeta Pasteur.

Foram medidos o pH, salinidade, concentração de oxigênio e de amônia, no início e no final de cada experimento. $\mathrm{O} \mathrm{pH}$ foi medido em pHmetro (Pradelski-digital) e a salinidade com refratômetro (ATAGO). A concentração de oxigênio foi estimada pelo método de Winkler modificado para pequenas amostras (Fox \& Wingfield, 1938) e a de amônia pelo método colorimétrico de Koroleff (1970).

Abaixo, encontra-se um resumo de todos os experimentos realizados. A denominação dos experimentos inclui o efluente utilizado (A ou B), o número seqüencial do experimento $\mathrm{e}$ indicação do estágio de desenvolvimento do animal, adultos (a) ou jovens (j).

\section{Efluente-A (amostrado no dia 25/11/91)}

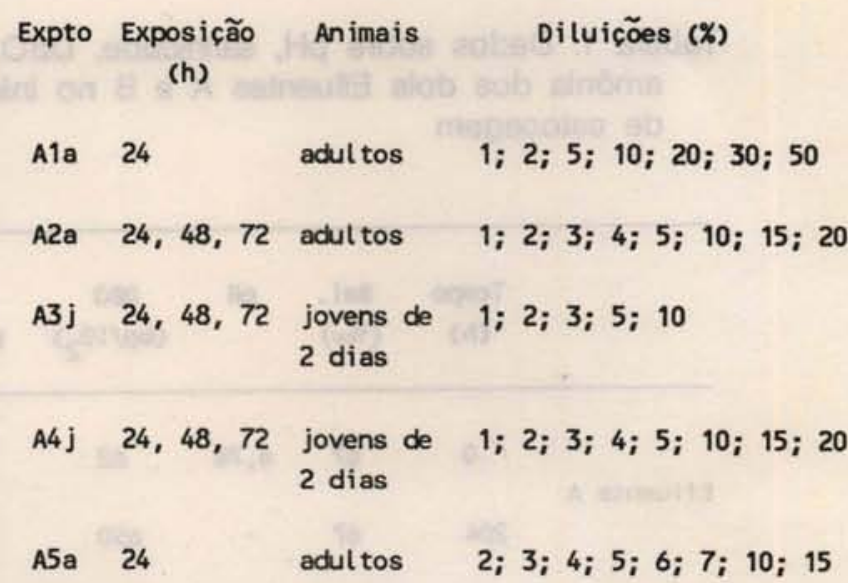

O Experimento-A1a foi realizado com o objetivo de determinar, de maneira preliminar, a faixa de concentração do Efluente-A a ser empregada nos testes subseqüentes. Esse experimento revelou que as concentraçōes a serem empregadas estavam entre $1 \%$ e $20 \%$. 
Os Experimentos-A2a e $\mathbf{A} 3 \mathbf{j}$, foram realizados concomitantemente, para determinar a CENO (concentração máxima do agente tóxico onde nāo é observado nenhum efeito estatisticamente significativo $\mathrm{em}$ relaçāo ao controle) e a CL(I)50 (concentração nominal do efluente onde ocorre a mortalidade de $50 \%$ dos indivíduos, num dado intervalo de tempo) para adultos e jovens de $P$. atlantica, respectivamente, após perfodos definidos de exposição às diversas concentraçōes.

Os Experimentos A4j e A5a, foram realizados para comparação com os Experimentos $\mathrm{A} 3 \mathrm{j}$ e $\mathrm{A} 2 \mathrm{a}$, respectivamente, a fim de verificar a variação da açāo tóxica do Efluente-A em função do tempo de armazenamento. Os Experimentos $\mathrm{A} 3 \mathrm{j}$ e $\mathrm{A} 4 \mathrm{j}$, ambos com jovens, foram realizados com diferença de 2 dias de armazenamento. Os Experimentos A2a e A5a, ambos com adultos, foram realizados com diferença de 6 dias de armazenamento do efluente $\mathrm{A}$.

\section{Efluente-B (amostrado no dia 06/01/92)}

Expto Exposição Animais Diluições (\%)

(h)

B1j 24,48

jovens de 0,$3 ; 0,6 ; 1,0 ; 1,3 ; 1,6$;

2 dias 2,$0 ; 2,3 ; 2,6 ; 3,0 ; 4,0$

B2j $24,48,72,96$ jovens de 0,$005 ; 0,01 ; 0,02 ; 0,03$; 2 dias 0,$05 ; 0,075 ; 0,10 ; 0,15$; 0,$20 ; 0,30$
Os experimentos com o Efluente-B, foram realizados apenas com jovens de $P$. atlantica com o objetivo de avaliar as possíveis diferenças de toxicidade entre os Efluentes-A e B, preparados em ocasiōes distintas. Iniciou-se por um experimento preliminar (B1j) para determinar a faixa de toxicidade a ser testada. O segundo experimento (B2j), para calcular a CENO e estimar a CL(I) 50 , serviu como base para comparação da magnitude da diferença de açāo entre as amostras A e B. Os experimentos B1j e B2j foram realizados com diferença de 6 dias de armazenamento do Efluente-B.

\section{Análises Estatísticas}

Foi estimada, para cada experimento, a CENO através do teste de Dunnett (Zar, 1984). Calculou-se também, para a maioria dos experimentos, a CL(I)50 utilizando o método de Hamilton et al. (1977). O programa para computador IBM-PC deste método foi gentilmente fornecido pela CETESB.

\section{Resultados}

Análises químicas de componentes gerais dos Efluentes A e B revelaram existir entre eles diferenças significativas de composiçāo. Na Tabela 1 encontram-se dados de salinidade, pH, DBO, DQO, óleos e graxas, sulfetos, fenóis e concentraçāo de amônia das duas amostras no início dos experimentos e após um determinado período de estocagem (204 horas para o efluente A e 240 horas para o efluente B).

Tabela 1. Dados sobre pH, salinidade, DBO, DQO, óleos e graxas, sulfetos, fenóis e amônia dos dois Efluentes A e B no início $(0 \mathrm{~h})$ e após um determinado período de estocagem

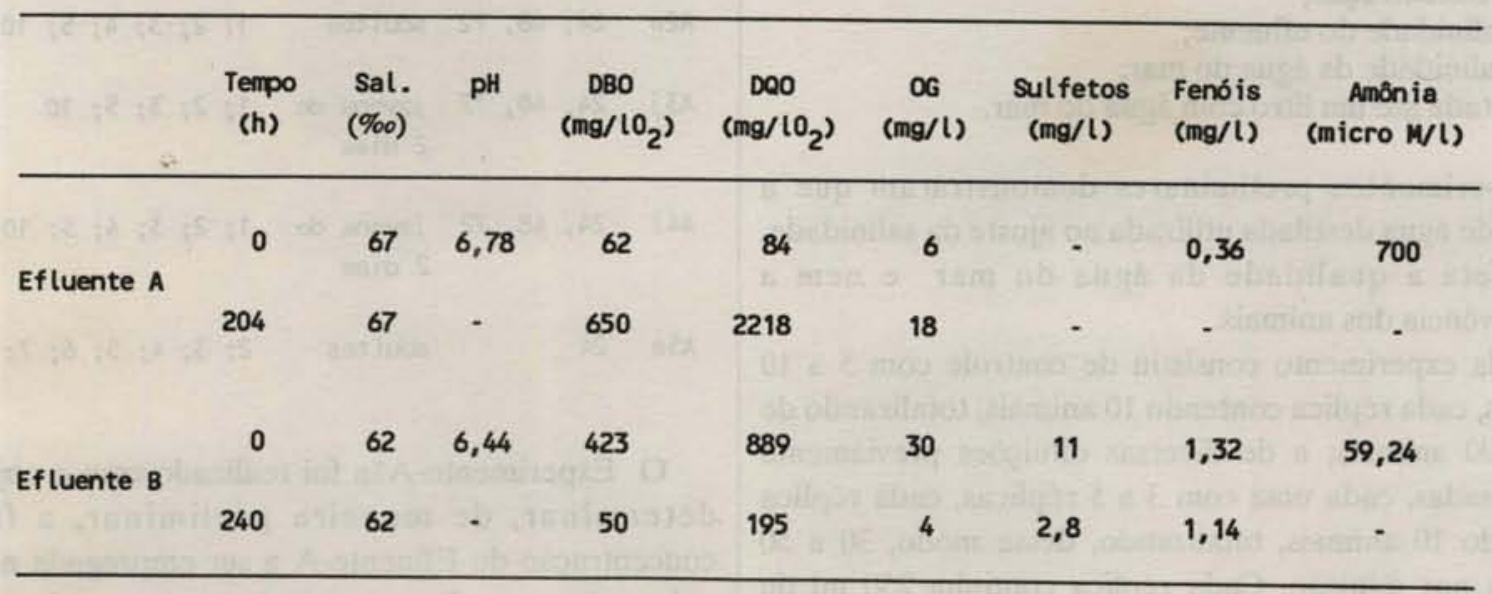


As porcentagens de sobrevivência tanto no controle quanto em cada concentração utilizada, os valores da CENO e da CL(I) 50 , para cada experimento, referente a cada amostra do efluente analisada, encontram-se na Tabela 2.

Tabela 2. Porcentagem de sobreviventes, CENO e CL(I)50 de $P$. atlantica em função do tempo e da concentração de exposição nos experimentos realizados com o Efluente-A (I) e B (II e III) (Concentração $0 \%=$ Controle)

I

Experimento Tempo

Sobreviventes (\%)

CENO CL(I)50

(h)

\begin{tabular}{rrrrrrrrrrrrrrrr}
\hline \multirow{2}{*}{ A2a } & 24 & 97 & 90 & 92 & 86 & 84 & 64 & - & - & 42 & 14 & 0 & $4-5$ & 7,38 \\
& 48 & 89 & 76 & 76 & 80 & 68 & 58 & - & - & 40 & 0 & 0 & $3-4$ & 6,61 \\
& 72 & 85 & 76 & 72 & 76 & 62 & 56 & - & - & 40 & 0 & 0 & $3-4$ & 6,10 \\
\multirow{2}{*}{ A3j } & 24 & 96 & 77 & 83 & 83 & - & 83 & - & - & 43 & - & - & $5-10$ & 8,86 \\
& 48 & 80 & 70 & 77 & 80 & - & 73 & - & - & 27 & - & - & $5-10$ & 7,07 \\
& 72 & 78 & 70 & 77 & 73 & - & 73 & - & - & 27 & - & - & $5-10$ & 7,07 \\
& 24 & 98 & 100 & 90 & 92 & 84 & 72 & - & - & 4 & 0 & 0 & $3-4$ & 5,63 \\
A4j & 48 & 96 & 100 & 84 & 86 & 80 & 68 & - & - & 2 & 0 & 0 & $1-2$ & 5,13 \\
& 72 & 95 & 92 & 70 & 72 & 72 & 58 & - & - & 0 & 0 & 0 & $1-2$ & 4,30 \\
& 24 & 94 & - & 88 & 72 & 14 & 8 & 0 & 0 & 0 & 0 & - & $2-3$ & 3,23
\end{tabular}

$\begin{array}{llllllllllll}\text { Concentraçoes (\%) } & 0 & 1 & 2 & 3 & 4 & 5 & 6 & 7 & 10 & 15 & 20\end{array}$

II

\begin{tabular}{|c|c|c|c|c|c|c|c|c|c|c|c|c|c|c|}
\hline far & 3aci ox & \multicolumn{2}{|c|}{$17 x^{2}, 2$} & \multicolumn{2}{|c|}{$\cos ^{2}+x^{2}$} & 20,19 & \multicolumn{2}{|c|}{ 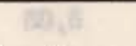 } & 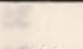 & \multirow{2}{*}{$3 x$} & \multirow{2}{*}{2} & \multirow{2}{*}{8} & \multirow{2}{*}{ CENO } & \multirow[b]{2}{*}{$\mathrm{CL}(\mathrm{I}) 50$} \\
\hline \multicolumn{2}{|c|}{ Experimento } & Tempo & - & & - & \multicolumn{3}{|c|}{ Sobreviventes } & $s(\%)$ & & & & & \\
\hline eif & 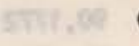 & (h) & 4,8 & coste & & 46,7 & ale & & 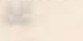 & 38 & 25 & 8 & (\%) & (\%) \\
\hline 4 & 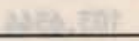 & $\mathrm{c}$ & + & 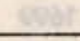 & & 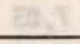 & 마단 & & 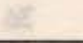 & 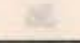 & 15 & 28 & & \\
\hline$\overline{7}$ & $x=80,35 ?$ & & - & $\operatorname{artin} 0$ & $\sqrt{4}=$ & 62,5 & Qut & & Ex & 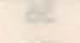 & 3 & 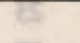 & 0,4 & \\
\hline B1 j & bis geat & 24 & 94 & 46 & 36 & 54 & 44 & 42 & 34 & 32 & $24 \quad 10$ & 0 & $<0,30$ & $<0,30$ \\
\hline & & 48 & 85 & 28 & 26 & 44 & 32 & 28 & 20 & 16 & $14 \quad 10$ & 0 & $<0,30$ & $<0,30$ \\
\hline Concent & traçöos & (\%) & 0 & 0,3 & 0,6 & 1,0 & 1,3 & 1,6 & 2,0 & 2,3 & $2,6 \quad 3,0$ & 4,0 & & \\
\hline
\end{tabular}

III

\begin{tabular}{ccccc}
\hline \multirow{2}{*}{ Experimento } & Tempo & Sobreviventes $(\%)$ & CENO & CL(I)50 \\
& $(h)$ & & $(\%)$ & $(\%)$ \\
\hline
\end{tabular}

B2j

$\begin{array}{llllllllllllll}24 & 100 & 100 & 98 & 98 & 94 & 100 & 92 & 90 & 98 & 98 & 88 & 0,2-0,3 & - \\ 48 & 99 & 94 & 96 & 96 & 92 & 96 & 90 & 88 & 92 & 90 & 84 & 0,2-0,3 & - \\ 72 & 98 & 94 & 94 & 94 & 88 & 96 & 88 & 84 & 80 & 80 & 64 & 0,1-0,15 & - \\ 96 & 95 & 94 & 98 & 90 & 84 & 94 & 86 & 78 & 74 & 76 & 64 & 0,075-0,1 & \sim 0,37\end{array}$

Concentraçoes (\%)

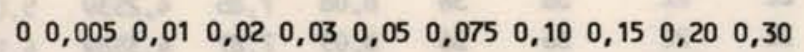


períodos de 24, 48 e 72 horas, respectivamente. Na Tabela 3 estăo os dados de temperatura, salinidade, $\mathrm{pH}$, concentração de oxigênio e de amônia no início e no final do experimento.

No Experimento-A3j, a CENO estava entre 5 a $10 \%$, nos três períodos. A CL(I)50 foi de $8,86 \%, 7,07 \%$ e novamente $7,07 \%$ após 24,48 e 72 horas de experimento, respectivamente. Dados referentes à temperatura, salinidade, $\mathrm{pH}$, concentraçāo de oxigênio e de amônia, no início e no final do experimento, estão na Tabela 4.

Em relação ao Experimento-A4j, a CENO após 24 horas estava entre 3 e $4 \%$; e após 48 e 72 horas entre 1 e $2 \%$. A CL(I) 50 foi de $5,63 \%, 5,13 \%$ e $4,30 \%$ apos 24,48 e 72 horas, respectivamente. Na Tabela 5 estão os dados de temperatura, salinidade, $\mathrm{pH}$, concentraçāo de oxigênio e de amônia, no início e no final do experimento.
Para o Experimento-A5a, a CENO estava entre 2 e $3 \%$ e a CL(I) 50 foi de $3,23 \%$ para a exposição de 24 horas. Na Tabela 6 estāo os dados de temperatura, salinidade, $\mathrm{pH}$, concentração de oxigênio e amônia para o início e o final do experimento.

Foram realizados com o Efluente-A, dois experimentos com jovens e dois com adultos, em condiçōes semelhantes, para verificar possiveis alteraçōes da toxicidade do efluente com o período de estocagem. Ressalta-se que o experimento com jovens (A4j) foi repetido dois dias após o anterior (A3j), mostrando que a CENO (24h) baixou de 5 a $10 \%$ para 3 a $4 \%$; o experimento com adultos (A5a) foi repetido seis dias após o anterior (A2a), mostrando que a CENO (24h) de 3 a $4 \%$ baixou para 2 a $3 \%$ (Tab. 2).

Tabela 3. Dados sobre temperatura, salinidade, $\mathrm{pH}$, concentração de oxigênio, concentração de amônia, no início $(0 \mathrm{~h})$ e no fim $(72 \mathrm{~h})$ do Experimento-A2a realizado com adultos de $P$. atlantica

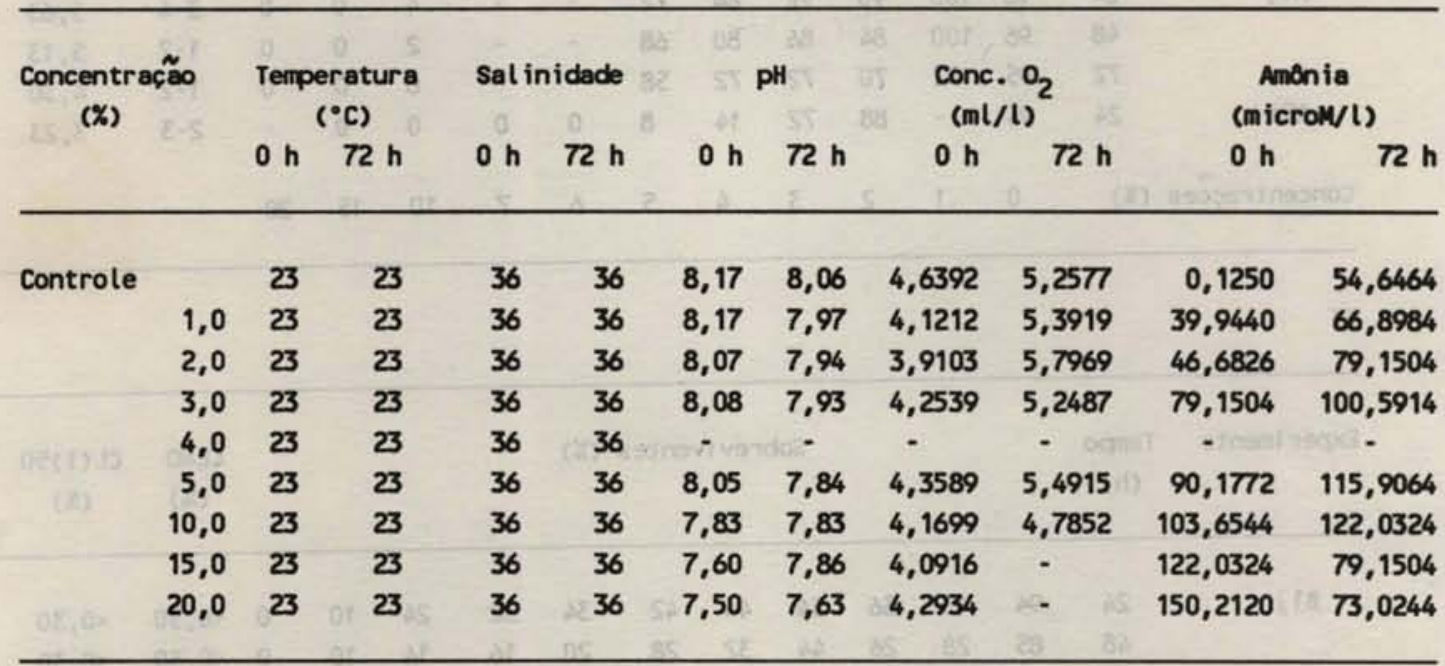

Tabela 4. Dados sobre temperatura, salinidade, pH, concentração de oxigênio, concentração de amônia, no início $(0 \mathrm{~h})$ e no fim $(72 \mathrm{~h})$ do Experimento-A3] realizado com jovens de $P$. atlantica

\begin{tabular}{|c|c|c|c|c|c|c|c|c|c|c|c|}
\hline \multirow{2}{*}{\multicolumn{2}{|c|}{$\begin{array}{l}\text { Concentração } \\
\text { (\%) }\end{array}$}} & \multicolumn{2}{|c|}{$\begin{array}{c}\text { Temperatura } \\
\left({ }^{\circ} \mathrm{C}\right)\end{array}$} & \multicolumn{2}{|c|}{ Sal inidade } & \multicolumn{2}{|c|}{$\mathrm{pH}$} & \multicolumn{2}{|c|}{$\begin{array}{l}\text { Conc. } \mathrm{O}_{2} \\
(\mathrm{ml} / \mathrm{t})^{2}\end{array}$} & \multicolumn{2}{|c|}{$\begin{array}{c}\text { Amônia } \\
\text { (microm/t) }\end{array}$} \\
\hline & & $\mathrm{Oh}$ & $72 \mathrm{~h}$ & $\mathrm{Oh}$ & $72 h$ & $\mathrm{Oh}$ & $72 \mathrm{~h}$ & $\mathrm{Oh}$ & $72 h$ & $\mathrm{Oh}$ & $72 \mathrm{~h}$ \\
\hline & $.065,0$ & 14 & 89 & (19? & $3 x$ & & & & & & \\
\hline \multirow{2}{*}{ Controle } & $9 \cdot 5 x^{2}$ & 22 & 22 & 36 & 36 & 8,17 & 8,10 & 4,6392 & 5,4296 & 0,1250 & 17,8904 \\
\hline & 1,0 & 22 & 22 & 36 & 36 & 8,17 & 8,00 & 4,1212 & 5,5293 & 39,9440 & 44,2322 \\
\hline \multirow[t]{4}{*}{$\sqrt{2}, 0-$} & 2,0 & 22 & 22 & 36 & 36 & 8,07 & 7,91 & 3,9103 & 5,5310 & 46,6826 & 69,3488 \\
\hline & 3,0 & 22 & 22 & 36 & 36 & 8,08 & 7,85 & 4,2539 & 5,0086 & 79,1504 & $\pi 7,9252$ \\
\hline & 5,0 & 22 & 22 & 36 & 36 & 8,05 & 7,87 & 4,3589 & 4,9767 & 90,1772 & 97,5284 \\
\hline & 10,0 & 22 & 22 & 36 & 36 & 7,83 & 7,20 & 4,1699 & 4,9219 & 103,6544 & 93,8528 \\
\hline
\end{tabular}


Tabela 5. Dados sobre temperatura, salinidade, pH, concentração de oxigênio, concentração de amônia, no inicio $(0 \mathrm{~h})$ e no fim $(72 \mathrm{~h})$ do Experimento-A4j realizado com jovens de $P$. atlantica

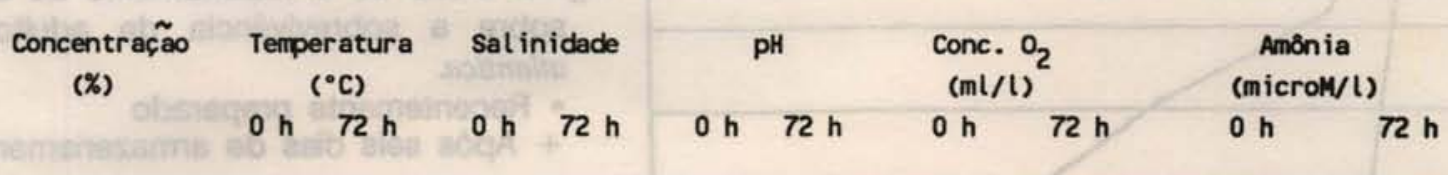

$\begin{array}{rrrlllllllll}\text { Controle } & & 23 & 23 & 38 & 38 & 8,16 & 8,10 & 5,7045 & 5,5670 & 0,1250 & 10,8088 \\ & 1,0 & 23 & 23 & 38 & 38 & 8,16 & 8,07 & 5,8727 & 5,8727 & 30,1424 & 31,0001 \\ & 2,0 & 23 & 23 & 38 & 38 & 8,16 & 8,07 & 5,6254 & 5,5911 & 54,6464 & 53,2987 \\ & 3,0 & 23 & 23 & 38 & 38 & 8,12 & 8,05 & 5,7290 & 5,3516 & 61,9976 & 64,8156 \\ & 4,0 & 23 & 23 & 38 & 38 & 8,11 & 8,04 & 5,8004 & 5,0454 & 74,2496 & 69,8389 \\ & 5,0 & 23 & 23 & 38 & 38 & 8,09 & 8,00 & 5,7080 & 5,2637 & 84,0512 & 88,5845 \\ 10,0 & 23 & 23 & 38 & 38 & 8,00 & 7,85 & 5,5918 & 5,3531 & 85,2764 & 117,1316 \\ 15,0 & 23 & 23 & 38 & 38 & 7,89 & 7,88 & 5,6330 & 5,3582 & 103,6544 & 138,2051 \\ 20,0 & 23 & 23 & 38 & 38 & 7,79 & 7,89 & 5,7374 & 5,4282 & 109,7804 & 146,0463\end{array}$

Tabela 6. Dados sobre temperatura, salinidade, $\mathrm{pH}$, concentração de oxigénio, concentração de amônia, no início $(0 \mathrm{~h})$ e no fim $(24 \mathrm{~h})$ do Experimento-A5a realizado com adultos de $P$. atlantica

\begin{tabular}{|c|c|c|c|c|c|c|c|c|c|c|c|}
\hline \multirow{2}{*}{\multicolumn{2}{|c|}{$\begin{array}{l}\text { Concentração } \\
\text { (\%) }\end{array}$}} & \multicolumn{2}{|c|}{$\begin{array}{c}\text { Temperatura } \\
\left({ }^{\circ} \mathrm{C}\right)\end{array}$} & \multicolumn{2}{|c|}{ Sal inidade } & \multirow{2}{*}{ o h } & $\mathrm{pH}$ & \multicolumn{2}{|c|}{$\begin{array}{l}\text { Conc. } \mathrm{O}_{2} \\
(\mathrm{ml} / \mathrm{l})\end{array}$} & \multicolumn{2}{|c|}{$\begin{array}{c}\text { Amônia } \\
\text { (microm/l) }\end{array}$} \\
\hline & & $\mathrm{Oh}$ & $24 \mathrm{~h}$ & $\mathrm{Oh}$ & $24 \mathrm{~h}$ & & $24 \mathrm{~h}$ & $\mathrm{Oh}$ & $24 \mathrm{~h}$ & $\mathrm{Oh}$ & $24 \mathrm{~h}$ \\
\hline \multirow[t]{6}{*}{ Controle } & & 23 & 23 & 36 & 36 & 8,23 & 8,13 & 6,1168 & 7,1134 & 0,1250 & 14,7907 \\
\hline & 2,0 & 23 & 23 & 36 & 36 & 8,20 & 8,02 & 6,1474 & 6,8343 & 56,4842 & 54,2789 \\
\hline & 3,0 & 23 & 23 & 36 & 36 & 8,18 & 8,00 & 5,8312 & 6,8259 & 77,9252 & 71,5542 \\
\hline & 4,0 & 23 & 23 & 36 & 36 & 8,14 & 7,91 & 5,9348 & 6,7925 & 79,1504 & 83,4386 \\
\hline & 5,0 & 23 & 23 & 36 & 36 & 8,12 & 7,95 & 5,7318 & 6,7615 & 97,5284 & 84,7863 \\
\hline & 6,0 & 23 & 23 & 36 & 36 & 8,10 & 7,83 & 5,9131 & 6,2891 & 109,7804 & 82,5810 \\
\hline 14:at & 7,0 & 23 & 23 & 36 & 36 & 8,05 & 7,88 & 5,7282 & 6,1374 & 115,9064 & 86,5016 \\
\hline $8+5 b$ ar & 10,0 & 23 & 23 & 36 & 36 & 7,98 & 7,86 & 5,9078 & 5,7017 & 115,9064 & 89,5646 \\
\hline & 15,0 & 23 & 23 & 36 & 36 & 7,88 & 7,71 & 6,0810 & 4,3632 & 122,0324 & 92,6276 \\
\hline
\end{tabular}

Na Figura 1 encontra-se a variação da porcentagem de sobrevivência de adultos de $P$. atlantica em função do tempo de estocagem do Efluente-A, constatada através dos Experimentos A2a e A5a.

\section{Efluente-B}

No Experimento-B1j os testes estatísticos revelaram que os valores da CENO e CL(I) 50 estavam abaixo de $0,30 \%$, que foi a concentração mínima empregada. Na
Tabela 7 estão os dados de temperatura, salinidade, $\mathrm{pH}$, concentraçāo de oxigênio e de amônia, para o início e o final do experimento.

Em relação ao Experimento-B2j, a CENO, após 24 e 48 horas estava entre 0,20 e $0,30 \%$, após 72 horas entre 0,10 e $0,15 \%$ e após 96 horas entre 0,075 e $0,10 \%$. A CL(I)50 após 96 horas foi estimada em torno de $0,37 \%$. Na Tabela 8 estão os dados referentes à temperatura, salinidade, $\mathrm{pH}$, concentraçāo de oxigênio e de amônia para o início e o final do experimento. 
(\%) Sobrevivência

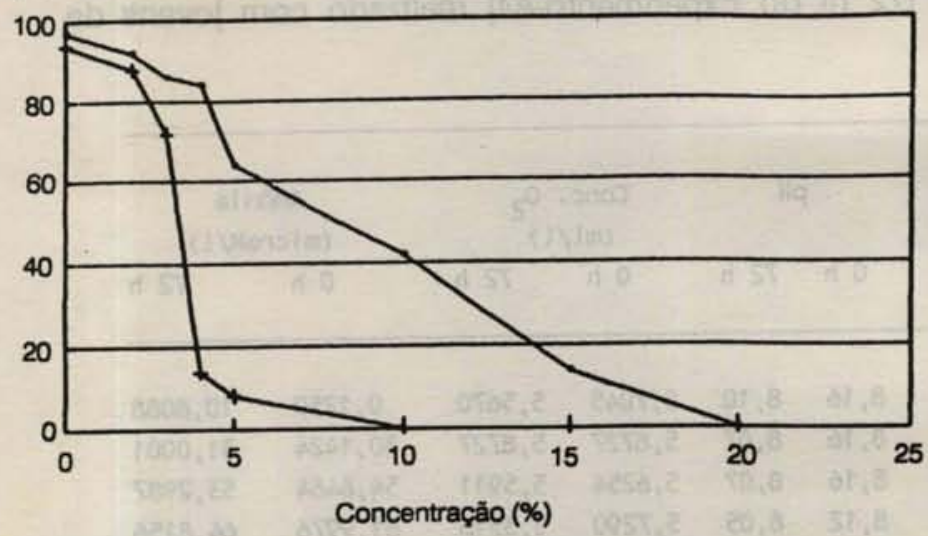

Fig. 1. Efeito do armazenamento do Efluente A sobre a sobrevivência de adultos de $P$. atlantica.

- Recentemente preparado

+ Após seis dias de armazenamento a $0^{\circ} \mathrm{C}$

Tabela 7. Dados sobre temperatura, salinidade, $\mathrm{pH}$, concentração de oxigênio, concentração de amônia, no inicio $(0 \mathrm{~h})$ e no fim $(48 \mathrm{~h})$ do Experimento-B1] realizado com jovens de $P$. atlantica

\begin{tabular}{|c|c|c|c|c|c|c|c|c|c|c|c|c|}
\hline \multicolumn{2}{|c|}{$\begin{array}{c}\text { Concentração } \\
\text { (\%) }\end{array}$} & \multicolumn{2}{|c|}{$\begin{array}{c}\text { Temperatura } \\
\left({ }^{\circ} \mathrm{C}\right)\end{array}$} & \multicolumn{2}{|c|}{ Sal inidade } & \multicolumn{2}{|c|}{ PH } & \multicolumn{2}{|c|}{$\begin{array}{c}\text { Conc. } \mathrm{O}_{2} \\
(\mathrm{ml} / \mathrm{l})^{2}\end{array}$} & 25 & \multicolumn{2}{|c|}{$\begin{array}{c}\text { Amônia } \\
\text { (microw/) }\end{array}$} \\
\hline & & O h & $48 h$ & 0 & $48 \mathrm{~h}$ & $\mathrm{Oh}$ & $48 \mathrm{~h}$ & $0 \mathrm{~h}$ & $48 \mathrm{~h}$ & & $\mathrm{Oh}$ & $48 h$ \\
\hline \multirow[t]{6}{*}{ Controle } & & 22 & 22 & 3 & 37 & 8,19 & 8,04 & 6,1856 & \multicolumn{2}{|l|}{4,7079} & 0,6151 & 3,3412 \\
\hline & 0,3 & 22 & 22 & 3 & 37 & 8,16 & 8,11 & 6,0444 & \multicolumn{2}{|l|}{4,6707} & 1,9628 & 1,9016 \\
\hline & 0,6 & 22 & 22 & 3 & 37 & 8,16 & 8,03 & 5,8312 & \multicolumn{2}{|l|}{4,8365} & 2,1466 & 4,2907 \\
\hline & 1,0 & 22 & 22 & 3 & 37 & 8,16 & 8,07 & 5,7633 & \multicolumn{2}{|l|}{4,4940} & 1,4727 & 4,3213 \\
\hline & 1,3 & 22 & 22 & 3 & 37 & 8,15 & 8,05 & 5,6632 & \multicolumn{2}{|l|}{4,5648} & 0,8601 & 4,7195 \\
\hline & 1,6 & 22 & 22 & 3 & 37 & 8,15 & 8,05 & 5,6738 & \multicolumn{2}{|l|}{4,2725} & 0,7376 & 6,4654 \\
\hline alnowas & 2,0 & 22 & 22 & 3 & 37 & 8,11 & 7,97 & 5,3531 & \multicolumn{2}{|l|}{4,4325} & 0,2475 & 6,7105 \\
\hline furveras & 2,3 & 22 & 22 & 3 & 37 & 8,11 & 8,01 & 5,4269 & \multicolumn{2}{|l|}{4,9804} & 0,1250 & 5,6384 \\
\hline & 2,6 & 22 & 22 & 3 & 37 & 8,10 & 8,03 & 5,0503 & \multicolumn{2}{|l|}{4,8098} & 0,2475 & 7,4150 \\
\hline & 3,0 & 22 & 22 & 3 & 37 & 8,08 & 7,94 & 5,2532 & \multicolumn{2}{|l|}{3,7768} & 0,6151 & 6,9249 \\
\hline & 4,0 & 22 & - & 3 & - & 8,04 & - & 4,7079 & \multicolumn{2}{|l|}{-} & 0,4313 & - \\
\hline Hit & 5,0 & 22 & - & 3 & - & 7,98 & - & 5,2202 & & 0,2475 & - \\
\hline ke & 7,0 & 22 & - & 3 & - & 7,90 & - & 4,9394 & \multicolumn{2}{|l|}{-} & 0,2475 & - \\
\hline${ }^{17}$ & 10,0 & 22 & - & 3 & $\cdot$ & 7,76 & - & 4,2882 & & 0,2475 & $\cdot$ \\
\hline$\sqrt{17}$ & 15,0 & 22 & - & 3 & - & 7,54 & - & 4,2903 & - & & 1,6565 & - \\
\hline
\end{tabular}

Tabela 8. Dados sobre temperatura, salinidade, $\mathrm{pH}$, concentração de oxigênio, concentração de amônia, no início $(0 \mathrm{~h})$ e no fim $(96 \mathrm{~h})$ do Experimento-B2j realizado com jovens de $P$. atlantica

\begin{tabular}{|c|c|c|c|c|c|c|c|c|c|c|c|}
\hline \multirow{2}{*}{\multicolumn{2}{|c|}{$\begin{array}{c}\text { Concentraçao } \\
\text { (x) }\end{array}$}} & \multicolumn{2}{|c|}{$\begin{array}{c}\text { Temperatura } \\
\left(^{\bullet} \mathrm{C}\right)\end{array}$} & \multicolumn{2}{|c|}{ Sal inidade } & \multicolumn{2}{|c|}{ pH } & \multicolumn{2}{|c|}{$\begin{array}{l}\text { Conc. } \mathrm{O}_{2} \\
(\mathrm{ml} / \mathrm{t})^{2}\end{array}$} & \multicolumn{2}{|c|}{$\begin{array}{c}\text { Amônia } \\
\text { (microM/I) }\end{array}$} \\
\hline & & $\mathrm{Oh}$ & $96 \mathrm{~h}$ & O h & $96 \mathrm{~h}$ & $\mathrm{Oh}$ & $96 \mathrm{~h}$ & $\mathrm{Oh}$ & $96 \mathrm{~h}$ & O h & $9 \mathrm{~h}$ \\
\hline Controle & & 23 & 23 & 38 & 38 & 8,15 & 8,04 & 5,3000 & 6,6293 & 0,1310 & 18,0288 \\
\hline & 0,005 & 23 & 23 & 38 & 38 & 8,14 & 8,05 & 5,4300 & 7,1535 & 0,1310 & 9,7976 \\
\hline & 0,010 & 23 & 23 & 38 & 38 & 8,15 & 8,02 & 5,4899 & 6,5554 & 0,1310 & 14,1140 \\
\hline & 0,020 & 23 & 23 & 38 & 38 & 8,14 & 8,06 & 5,4906 & 6,9292 & 0,0306 & 8,0912 \\
\hline ST rog & 0,030 & 23 & 23 & 38 & 38 & 8,15 & 8,07 & 5,6264 & 6,8132 & 0,4322 & 17,8280 \\
\hline 3. टा0द्व & 0,050 & 23 & 23 & 38 & 38 & 8,16 & 8,04 & 5,4705 & 5,8667 & 0,4322 & 31,8812 \\
\hline andot & 0,075 & 23 & 23 & 38 & 38 & 8,16 & 8,04 & 5,4902 & 6,9581 & 0,7333 & 37,2014 \\
\hline & 0,100 & 23 & 23 & 38 & 38 & 8,16 & 8,04 & 5,4973 & 6,4302 & 0,7333 & 18,6311 \\
\hline & 0,150 & 23 & 23 & 38 & 38 & 8,15 & 8,10 & 5,3653 & 6,4651 & 0,9341 & 17,4265 \\
\hline & 0,200 & 23 & 23 & 38 & 38 & 8,16 & 8,07 & 5,7617 & 6,9940 & 0,9341 & 20,6387 \\
\hline & 0,300 & 23 & 23 & 38 & 38 & 8,16 & 8,10 & 5,3333 & 4,8304 & 1,1348 & 3,3733 \\
\hline
\end{tabular}




\section{Discussão}

Várias espécies de misidáceos vêm sendo empregadas rotineiramente em estudos de toxicidade por diversos autores com bastante sucesso (Nimmo et al., 1977; Breteler et al., 1982; Lussier et al., 1985; Anderson et al., 1988). Os resultados obtidos neste trabalho demonstraram ser $P$. atlantica uma espécie bastante adequada para bioensaios de toxicidade aguda. A facilidade de coleta, estocagem, manuseio e a taxa de sobrevivência nas réplicas controle apoiam essa afirmativa. Além disso, os animais estāo disponíveis na região o ano todo e, aparentemente, também há disponibilidade de jovens no decorrer de todo esse período.

O efluente utilizado neste trabalho foi uma simulação do efluente real e os experimentos realizados são de caráter preliminar. Os resultados obtidos, no entanto, demonstram alguns aspectos importantes da sua toxicidade, fornecem subsídios para a avaliação do método de tratamento e indicam as necessidades de acompanhamento das variaçōes em sua composição química e de monitorização de possíveis efeitos ambientais decorrentes do lançamento desse material no mar.

Os valores da CENO, apesar de serem usualmente obtidos em testes de toxicidade crônica, podem também ser calculados a partir de testes de toxicidade aguda (Martin et al., 1988). Esses valores foram estimados para se inferir sua ordem de grandeza, visando experimentos futuros.

Os resultados obtidos nos experimento com o Efluente-A para jovens (A3j), realizado concomitantemente com o de adultos (A2a), demonstraram que os valores da CENO foram maiores para os jovens nos três períodos testados (Tab. 2). Esses resultados sugerem ser os jovens mais resistentes que os adultos, pelo menos a essa amostra do efluente. Diferenças na resistência a poluentes dependendo do estágio do desenvolvimento são conhecidas em outros animais, se bem que nem sempre facilmente explicáveis (Johns \& Pechenik, 1980; Johns \& Miller, 1982). Neste trabalho, a diferença encontrada também poderia ser devida a outros fatores, tais como a idade nāo homogênea dos adultos ou ainda ao estresse decorrente do transporte e manuseio. Os adultos, apesar da alta sobrevivência nos frascos controle e nos aquários onde foram mantidos, sofreram o processo de coleta e transporte. Por outro lado, os jovens empregados foram aqueles liberados do marsúpio no laboratório, o que provavelmente é um fator positivo.

O efluente tem composiçāo extremamente complexa e durante a estocagem ocorreram alterações na sua composição e no seu potencial tóxico. Experimentos repetidos em dias diferentes, tanto com jovens (A3j e A4j) quanto com adultos (A2a e A5a), demonstraram que a CENO e a CLI(50), para um mesmo período de exposição, diminuiram com a estocagem do Efluente-A refletindo um aumento de toxicidade do mesmo (Tab. 2). Note-se que a diminuiçâo desses valores foi semelhante tanto para jovens quanto para adultos. Examinando-se os resultados da análise química do efluente (Tab. 1) verifica-se que, no caso do Efluente-A, a DBO e DQO sofreram aumento com a estocagem refletindo possíveis alteraçōes químicas. Apesar da natureza dessas alterações não ser conhecida estas foram, provavelmente, responsáveis pelo aumento da toxicidade da amostra. A alta sobrevivência obtida nas 10 réplicas dos frascos controle, nos quatro experimentos, torna remota a possibilidade dessas diferenças da CENO e CL(I) 50 terem sido ocasionadas por qualquer fator relacionado com o estado fisiológico dos animais.

De modo geral, os experimentos com o Efluente-A demonstraram que, decorridas 24 horas de exposição, a concentração máxima onde não é observado efeito estatisticamente significativo na mortalidade de $P$. atlantica está entre 2 a $5 \%$. A partir dessas concentrações, a mortalidade desses animais já é estatisticamente diferente da do grupo controle. Por outro lado, aumentando-se o período de exposição para além das 24 horas, a CENO tem a tendência a diminuir ainda mais. Pode-se notar através dos experimentos A2a e A4j que, tanto para jovens quanto para adultos, a CENO sofreu uma diminuição da mesma ordem de grandeza após $\mathbf{4 8}$ horas, em relação aos valores obtidos para 24 horas, estabilizando-se após esse período (Tab. 2). Com os experimentos realizados, não se pode inferir se a tendência à estabilização da CENO mantém-se ou não para períodos mais longos de tempo. Aumento da mortalidade e, portanto, diminuição dos valores da CENO e CL(I) 50 são relativamente comuns com o aumento do período de exposição, provavelmente devido à ação prolongada das substâncias tóxicas presentes no meio (Gentile et al., 1982; Reish et al., 1989). Entretanto, o oposto também pode ocorrer quando as substâncias presentes no composto tóxico se degradam com o tempo (Reish et al., 1989).

Os testes realizados com o Efluente-B, indicaram que este apresenta um potencial tóxico bastante diferente do apresentado pela amostra A. Após um experimento preliminar (B1j), sempre necessário para se estabelecer o espectro de ação da substância a ser avaliada, chegou-se à conclusão de que a concentração máxima onde nenhum efeito sobre a sobrevivência dos animais era observado, estava abaixo de $0,3 \%$. No experimento seguinte (B2j) observou-se que a CENO apos 24 horas estava entre 0,2 a $0,3 \%$, correspondendo ao esperado pelo experimento preliminar. Esse valor é cerca de 13 vezes mais baixo do que o correspondente para exemplares jovens expostos ao Efluente-A, pelo mesmo período (Tab. 2). Após 72 horas a CENO caiu para 0,1 a $0,15 \%$, sendo cerca de 10 vezes 
mais baixa que a correspondente, para o mesmo período, no Experimento-A4j com o Efluente-A.

Por outro lado, a CL(I) 50 , ou seja, a concentraçáo mínima suficiente para causar a morte de $50 \%$ dos indivíduos, é uma medida do efeito drástico de um tóxico, do ponto de vista ecológico, usualmente calculada em experimentos de toxicidade aguda (Parsons et al., 1984). Comparando-se os resultados da CLI(50) obtidos para adultos no Experimento-A2a com aqueles do Experimento - A3j, realizado no mesmo dia com jovens da espécie, verificou-se serem os jovens um pouco mais resistentes que os adultos. Com a estocagem do Efluente-A, os valores da CL(I) 50 , naturalmente acompanhando o já comentado em relação aos valores da CENO, foram mais baixos tanto para jovens quanto para adultos, indicando aumento da toxicidade com a estocagem, pelo menos no caso específico dessa amostra.

O Efluente-B demonstrou efeito tóxico ainda mais pronunciado do que o Efluente-A, o que poderia ser correlacionado com os seus valores iniciais bastante mais elevados de DBO, DQO e OG (Tab. 1). Entretanto, ao contrário do ocorrido com o Efluente-A, esses valores decairam de forma pronunciada com a estocagem (Tab.1). Essa diminuição não esperada da concentração de compostos químicos provavelmente resultaram em diminuição da toxicidade do Efluente-B. Por esse motivo, as concentraçōes utilizadas no segundo experimento (B2j) com o Efluente-B, estabelecidas a partir do primeiro experimento (B1j) realizado 6 dias antes, foram ligeiramente aquém das necessárias para estimar com precisão a CL(I)50. Entretanto, foi possível inferir que esta se encontrava em torno de $0,37 \%$. Esse valor indica ser o efeito tóxico do Efluente-B, cerca de 10 vezes mais potente que o da amostra anterior, concordando com os resultados obtidos para a CENO.

Os valores encontrados, tanto para o Efluente-A quanto para o B, demonstram a alta toxicidade de ambas as amostras, principalmente se levarmos em consideração de que alteraçōes comportamentais e fisiológicas de animais expostos a substâncias tóxicas podem ocorrer em concentraçōes até $75 \%$ menores do que a CL(I) 50 (Diamond et al., 1990; Little \& Finger, 1990).

As razōes das diferenças de toxicidade entre os dois efluentes e as transformaçōes químicas que ocorrem com a estocagem são provavelmente, de uma extrema complexidade. Parece também que essas transformaçōes podem ser, inclusive, de naturezas opostas. $\mathrm{O}$ grande número de componentes existentes nos efluentes, bem como a não reprodutividade do tratamento poderiam ser responsáveis por essa instabilidade. Seriam necessárias análises químicas detalhadas, para que se pudesse ter uma idéia dos processos envolvidos, o que está além dos objetivos deste trabalho. Além disso, devido às contínuas alteraçōes dos efluentes testados, possíveis modificaçōes de sua toxicidade no decorrer dos experimentos são de difícil controle.

Muitos efluentes industriais são misturas complexas que variam constantemente de composição. $O$ acompanhamento das transformaçōes químicas e do destino das substâncias tóxicas no ecossistema são muito importantes para uma monitorização ambiental eficiente (Walker, 1990). No entanto, as análises de muitas das transformaçōes envolvidas são de difícil realizaçāo, além de serem custosas (Pequegnat \& Wastler, 1980; Schaeffer et al., 1982). Por esses motivos, a maneira mais eficaz de monitorizar os efeitos das transformaçōes de misturas complexas sobre o ambiente é através da resposta dos organismos às mesmas (Schalie et al., 1979; Hose et al., 1987; Svanberg \& Renberg, 1988; Clarke et al., 1990).

Os altos DBO e DQO iniciais do Efluente-B fizeram com que a concentração de oxigênio diminuisse significativamente nas concentraçōes mais altas, logo no início do experimento B1j (Tab. 7). Este efeito pôde ser observado porque as concentraçōes utilizadas no experimento $\mathrm{B} 1 \mathrm{j}$, baseadas no que seria esperado pelos testes com o Efluente-A, foram muito altas quando levada em conta a toxicidade bem mais pronunciada do Efluente-B. Nos demais experimentos, as concentraçōes nāo foram suficientemente elevadas para causarem efeito semelhante. Diminuições na concentração do oxigênio ambiental são causadas por vários poluentes com sérias conseqüências para a fauna (Umezawa \& Komatsu, 1980; Kramer, 1987). Se devido a sua alta densidade o efluente tiver dificuldade em diluir-se totalmente no corpo d'água, acumulando-se no fundo, a comunidade bentônica poderá ser bastante afetada.

Por outro lado, em vários experimentos com o Efluente-A (Tabs 3, 4 e 6) e no experimento B2j com o Efluente-B (Tab. 8), observou- se um ligeiro aumento da concentraçāo de oxigênio, no decorrer dos mesmos. As razões desse aumento não podem ser prontamente explicadas. No entanto, pode-se afirmar que este nāo foi devido à ação do efluente, pois ocorreu também nos frascos controle. Além disso, mesmo considerando-se um possível erro nessas mediçōes, a concentraçāo de oxigênio não deve ter afetado significativamente a mortalidade dos animais pois as diferenças de medida entre o inf́cio e o final dos experimentos sāo de mesma ordem de grandeza, tanto nos frascos controle quanto nos experimentais, tendo sido a sobrevivência nos frascos controle bastante elevada.

Além da alta toxicidade, tanto da amostra A quanto da $\mathrm{B}$, devido à presença de compostos químicos diversos não identificados, o efluente apresenta outras características potencialmente prejudiciais para o ambiente tais como cor escura, odor ofensivo, alta salinidade, alta acidez, alta concentração de amônia e baixa concentração de oxigênio. Apesar do efluente ser naturalmente hipersalino, nos experimentos realizados decidiu-se eliminar o efeito da 
salinidade sobre os animais e testar apenas o dos compostos tóxicos do mesmo, partindo-se do princípio que a alteração da concentração de sais no ambiente pode não afetar uma espécie em particular mas, certamente, alterará a biota local.

$\mathrm{O}$ pH do efluente, por outro lado, é mais ácido que o da água do mar (Tab. 1). Por esse motivo, o pH tem a nítida tendência a diminuir das menores para as maiores concentraçōes testadas e com o tempo de exposição (Tabs 3 a 8). Mesmo considerando-se as propriedades de tamponamento da água do mar, um despejo contínuo poderia alterar os valores de $\mathrm{pH}$, mesmo que apenas localmente. Alteraçōes do $\mathrm{pH}$ podem afetar diversos processos biológicos dos organismos marinhos. Os efeitos desta variaçāo podem ser consideráveis tendo em vista a alta estabilidade do $\mathrm{pH}$ da água do mar à qual estão adaptados (Cleveland et al.,1986). Por outro lado, a transparência da água é outro fator ambiental importante pois esta é fundamental para a fotossíntese. A cor escura do efluente pode impedir a penetração de luz, pelo menos em caráter local, com conseqüências sobre a sobrevivência e a produção dos organismos fitoplanctônicos que constituem a base da cadeia trófica. Ambos os efluentes possuem concentração elevada de amônia e odor altamente ofensivo. Apesar de que no mar a concentração de amônia é fator limitante para a produção primária, concentraçōes da mesma acima dos nf́veis toleráveis são prejudiciais. Muitos organismos aquáticos utilizam a olfaçāo e a quimiorrecepçāo para a procura de alimento, para a reprodução e percepção do ambiente; o odor e os efeitos de substâncias tóxicas podem causar sérios danos aos organismos pela alteraçāo dessas atividades (Dillon \& Gibson, 1985; Lemly \& Smith, 1986). Para uma efetiva monitorização ambiental todos os possíveis efeitos, tais como os aqui discutidos, devem ser levados em consideração.

\section{Conclusões}

De um modo geral, dentro dos objetivos deste trabalho, os experimentos realizados forneceram indicações bastante claras sobre o potencial tóxico das amostras do efluente testadas e, principalmente, de sua variabilidade.

Em relaçāo ao Efluente-A, concentraçōes entre $1 \%$ a $5 \%$ podem começar a aumentar significativamente a taxa de mortalidade de $P$. atlantica, em curto intervalo de tempo. No caso do Efluente-B, concentraçōes tão baixas quanto $0,075 \%$ (em período de 96 horas de exposição) estão no limite máximo de concentração a nāo afetar a sobrevivência dos indivíduos jovens da mesma espécie. Os experimentos demonstram consistentemente que a segunda amostra do efluente é cerca de 10 vezes mais potente do ponto de vista toxicologico que a primeira amostra.

Além da variabilidade amostral, há indicaçāo de que a estocagem pode alterar a ação tóxica do efluente e que esta alteração também depende da amostra. No caso do Efluente-A, os resultados demonstraram claramente haver um aumento da toxicidade com a estocagem. Por outro lado, em relação ao Efluente-B, há indicaçōes de que a toxicidade diminuiu com o tempo.

O efluente é uma mistura de odor ofensivo, com alta salinidade, alta concentração de amônia, baixo pH, oxigênio e transparência, fatores que não foram estudados neste trabalho mas que potencialmente podem causar efeitos deletérios para o meio ambiente.

Mais estudos sobre os efeitos ambientais do efluente ainda são necessários. Os resultados obtidos através deste trabalho preliminar, no entanto, são suficientes para indicar a necessidade de um programa de monitorização constante e rigoroso para o lançamento deste material no ambiente marinho.

\section{Agradecimentos}

Agradecemos ao Instituto Oceanográfico da USP e à FUNDESPA - Fundação de Estudos e Pesquisas Aquáticas, pelo apoio concedido, pelas instalaçōes e coordenação da parte financeira do projeto. À PETROBRÁS, pelo financiamento do trabalho, pelo fornecimento das amostras do efluente e pela autorizaçäo em divulgar os resultados. À Profa Dra Yoko Wakabara, pela identificaçāo dos misidáceos. Às Profas Dras Elizabeth Aidar e Eduinetty Ceci P. M. de Sousa, pela leitura do manuscrito. A todo o pessoal do nosso laboratório pelo inestimável auxilio.

\section{Referências bibliográficas}

AMERICAN PUBLIC HEALTH ASSOCIATION 1985. Standard Methods for the Examination of Water and Wastewater. $16^{\text {th }}$ ed. Amer. Publ. Health Asson. 1268p.

ANDERSON, B. S.; HUNT, J. W.; MARTINS, M.; TURPEN, S. L. \& PALMER, F. H. 1988. Protocol development: Reference toxicant and initial complex effluent testing. Marine Bioassay Project, Third Report. Division of water quality report $\mathrm{n}^{2}$ 88-7, 154p.

BELLAN, G. 1981. Manual of methods in aquatic environment research. Part 7. Selected bioassays for the Mediterranean. FAO Fish. Tech. Pap., $\mathbf{n}^{\mathbf{e}}$ 208, 31p. 
BRETELER, R. J.; WILLIAMS, J. W. \& BUHL, R. L. 1982. Measurement of chronic toxicity using the opossum shrimp Mysidopsis bahia. Hydrobiologia, 93:189-194.

CAPUZZO, J. M.; MOORE, M. N. \& WIDDOWS, J. 1988. Effects of toxic chemicals in the marine environment: predictions of impacts from laboratory studies. Aquat. Toxicol., 11:303-311.

CLARKE, S. M.; BARRICK, C. W. \& SAMOILOFF, M. R. 1990. A bioassessment battery for use in an industrial setting: a new management approach. Int. J. Toxicity Assessm., 5:153-166.

CLEVELAND, L.; LITTLE, E. E.; HAMILTON, S. J.; BUCKLER, D. R. \& HUNN, J. B. 1986. Interactive toxicity of aluminum and acidity to early life stages of brook trout. Trans. Am. Fish. Soc., 115:610-620.

CRADDOCK, D. R. 1977. Acute toxic effects of petroleum on Arctic and Subarctic marine organisms. In: Effects of petroleum on Arctic and Subarctic marine environments and organisms. Vol. II. Biological effects. 1977. D. C. Malins ed. New York, Academic Press Inc, 500p.

DIAMOND, J. M.; MAROLYN, J. P. \& GRUBER, D. 1990. Rapid detection of sublethal toxicity using fish ventilatory behavior. Environ. Toxicol. Chem., 9:3-11.

DILLON, T. M. \& GIBSON, A. B. 1985. Bioaccumulation and effects on reproduction in aquatic organisms: an assessment of the current literature. Miscellaneous paper D-85-2, Department of the Army, US Army Corps of Engineers. $34 \mathrm{p}$.

EVANS, M. S.; BATHELT, R. W. \& RICE, C. P. 1982. PCBs and other toxicants in Mysis relicta. Hydrobiologia, 93:205-215.

FOX, M. \& WINGFIELD, C. A. 1938. A portable apparatus for the determination of oxygen dissolved in a small volume of water. J. exp. Biol., 15:437-445.

GENTILE, S. M.; GENTILE, J. H.; WALKER, J. \& HELTSHE, J. F. 1982. Chronic effects of cadmium on two species of mysid shrimp: Mysidopsis bahia and Mysidopsis bigelowi. Hydrobiologia, 93:195204.

HAMILTON, M. A.; RUSSO, R. C. \& THURSTON, R. V.1977. Trimmed Spearman-Karber method for estimating median lethal concentrations in toxicity bioassays. Environ. Sci. Tech., 11(7):714-719. Correction: 12(4):417 (1978).
HOSE, J. E.; CROSS, J. N.; SMITH, S. G. \& DARIO, D. 1987. Elevated circulating erythrocyte micronuclei in fishes from contaminated sites off Southern California. Mar. Environ. Res., 22:167-176.

JOHNS, D. M. \& MILLER, D. C. 1982. The use of bioenergetics to investigate the mechanisms of pollutant toxicity in crustaceans larvae. In: Physiological mechanisms of marine pollutant toxicity. Verneberg, W. B.; Calabrese, A.; Thurberg, F. P. \& Verneberg, F. J. eds New York, Academic Press, p. 261-288.

\& PECHENIK, J. A. 1980. Influence of the water- accomodated fraction of $n^{2} 2$ fuel oil on energetics of Cancer irroraturus larvae. Mar. Biol., 55:247-254.

KOROLEFF, F. 1970. Direct determination of ammonia in natural waters as indophenol blue. Cons. int. Explor. Mer, Information on techniques and methods for sea water analysis., $n^{2} 3$.

KRAMER, D. L. 1987. Dissolved oxygen and fish behavior. Environ. Biol. Fishes, 18(2):81-92.

LEMLY, A. D. \& SMITH, J. E. 1986. A behavioral assay for assessing effects of pollutants on fish chemoreception. Ecotoxicol. Environ. Safety, 11:210-218.

LITTLE, E. W. \& FINGER, S. E. 1990. Swimming behavior as an indicator of sublethal toxicity in fish. Environ. Toxicol. Chem., 9:13-19.

LUSSIER, S. M.; GENTILE, J. M. \& WALKER, J. 1985. Acute and chronic effects of heavy metals and cyanide on Mysidopsis bahia (Crustacea: Mysidacea). Aquat. Toxicol., 7:25-35.

MARTIN, M.; ANDERSON, B. S.; HUNT, J. W. \& TURPEN, S. L. 1988. Mysid Tests. State Water Resources Control Board. Marine Bioassay Project Third Report. Division of Water Quality. $\mathbf{n}^{9}$ 88-7, p. 68-89.

NIMMO, D. R.; BAHNER, L. H.; RIGBY, R. A.; SHEPPARD, J. M. \& WILSON Jr, A. J. 1977. Mysidopsis bahia: an estuarine species suitable for life-cycle toxicity tests to determine the effects of a pollutant. In: Aquatic Toxicology and Hazard Evaluation, ASTM STP 634. Mayer, F. L. \& Hamelink, J. L. eds American Society for Testing and Materials, p. 109-116.

\& HAMAKER, T. L. 1982. Mysids in toxicity testing - a review. Hydrobiologia, 93:171-178. 
PARSONS, T. R.; TAKAHASHI, M. \& HARGRAVE, B. 1984. Biological Processes. Oxford, Pergamon Press, 330p.

PEQUEGNAT, W. E. \& WASTLER, T. A. 1980. Field bioassays for early detection of chronic impacts of chemical wastes upon marine organisms. Helgolander Meeresunters, 33: 531-545.

REISH, D. J.; ASATO, S. L. \& LEMAY, J. A. 1989. The effect of cadmium and DDT on the survival and regeneration in the amphinomid polychaete Eurythoe complanata. VII Symp. Int. Biol. Mar., 107-111.

SCHAEFFER, D.; KERSTER, H. W. \& JANARDAN, K. G. 1982. Monitoring toxics by group testing. Environ. Manag., 6:467-469.

SCHALIE, W. H. V. D.; DICKSON, K. L.; WESTLAKE, G. F. \& CAIRNS Jr, J. 1979. Fish bioassay monitoring of waste effluents. Environ. Manag., 3:217-235.

SVANBERG, O. \& RENBERG, L. 1988. Biological chemical characterization of effluents for the evaluation of the potential impact on the aquatic environment. In: Organic micropollutants in the aquatic environment. Angeletti, G. \& Bjorseth, A. eds Proc. $5^{\text {th }}$ European Symposium, October 20-22, 1987, Italy, Kluwer Academic Publishers, p. 244-255.
UMEZAWA, S. \& KOMATSU, K. 1980. Effect of turbidity on the toxicity of surfactants to the medaka, Oryzias latipes. Rep. Usa Mar. Biol. Inst., 2:1-9.

WALKER, J. D. 1990. Chemical fate, bioconcentration, and environmental effects testing: proposed testing and decision criteria. Int. J. Toxic. Assessm., 5:103134.

WARD, G. S. \& PARRISH, P. R. 1982. Manual of Methods in Aquatic Environment Research. Part 6. Toxicity tests. FAO Fish. Tech. Pap. $n^{2} 185,23 p$.

ZAR, J. H. 1984. Biostatistical analysis. Englewood Cliffs, Prentice-Hall. 718p.

(Manuscrito recebido 15 julho 1993; revisto 30 novembro 1994; aceito 22 dezembro 1994) 